\title{
Voluntary reporting to assess symptom burden among Yemeni cancer patients: common symptoms are frequently missed
}

\author{
Samy A Alsirafy, MBBCh, MSc, MD, DipPallMed, ${ }^{a}$ Khalil A Al-Alimi, MBBCh, MSc, ${ }^{b}$

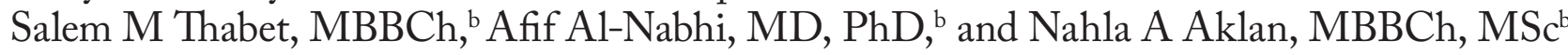 \\ aPalliative Medicine Unit, Kasr A1-Ainy Center of Clinical Oncology \& Nuclear Medicine, Kasr Al-Ainy School of Medicine, Cairo

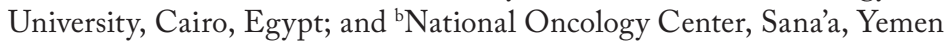

\begin{abstract}
Background Adequate symptom assessment is necessary to relieve the high symptom burden experienced by cancer patients. However, health care professionals may depend only on patient's voluntary reporting (VR) to assess symptoms and therefore some symptoms may be missed.
\end{abstract}

Objective To assess the symptom burden experienced by Yemeni cancer patients by using VR and systematic assessment (SA). Methods 50 cancer patients were asked an open question to voluntarily report their symptoms. This was followed by an SA of a list of 20 common physical symptoms that was drawn up based on the literature.

Results From 375 symptom entries related to the 20 symptoms, VR accounted for 66 entries (18\%) and SA for 309 (82\%). The mean number of VR symptoms/patient was 1.3, and the mean number of VR plus SA symptoms was $7.5(P<.001)$. In all, $74 \%$ of VR symptoms and $57 \%$ of SA symptoms were moderate or severe. For each symptom, the percentage of patients who experienced it and did not report it voluntarily (missed) was 100\% for bleeding, constipation, early satiety, hoarseness, taste changes, and weight loss. These were followed by anorexia (97\%), skin symptoms $(92 \%)$, dry mouth $(91 \%)$, edema $(89 \%)$, dyspnea $(88 \%)$, sore mouth $(88 \%)$, fatigue/weakness $(85 \%)$, diarrhea $(80 \%)$, dysphagia $(80 \%)$, nausea $(76 \%)$, cough $(75 \%)$, urinary symptoms $(75 \%)$, vomiting $(62 \%)$, and pain (18\%). Pain was the most common voluntarily reported symptom (56\% of patients), the most commonly distressing $(42 \%)$, and the least under-reported (18\%).

Limitations Relatively small sample size; the SA included only 20 symptoms.

Conclusions SA of symptoms yields a more accurate estimation of symptom burden than does VR. As with many developing countries where the majority of cancer patients present at an incurable disease stage, Yemeni cancer patients suffer a high symptom burden, especially pain.

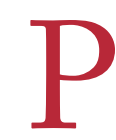
atients with cancer experience a high symptom burden. There is a large body of evidence documenting this fact in patients with advanced incurable cancer. ${ }^{1}$ However, this symptom burden is not limited to patients with terminal cancer and extends to involve the entire spectrum of the cancer trajectory. Even newly diagnosed patients with early cancer may experience a significant load of symptoms that are comparable with those in patients with advanced cancer., ${ }^{2,3}$ For example, almost two-thirds of cancer patients who receive anticancer treatment, as well as those with advanced, metastatic, or terminal cancer, have pain. ${ }^{4}$

Adequate multidimensional symptom control is necessary because uncontrolled symptoms have a negative impact on the quality of life of cancer patients. ${ }^{5}$ An important initial step in controlling symptoms in cancer patients is early identification and thorough assessment. ${ }^{6}$

Yemeni cancer patients are treated mainly at the National Oncology Center of Yemen in Sana'a, which is the main cancer care center in the country. The center receives about 5,000 new cancer patients each year, of whom the majority present initially in an advanced stage. ${ }^{7,8}$ Certainly, adequate symptom assessment and control is needed to improve the quality of life of these patients. ${ }^{6}$ However, with limited resources and overcrowded service at the center, it is not an uncommon practice for health care professionals to depend on patients' voluntary reporting (VR) for symptom assessments. However, previous studies have shown that symptoms are frequently missed when VR alone is used for assessment. ${ }^{9-11}$

In this article, we describe the symptom burden

Accepted for publication September 19, 2015. Correspondence: Samy A Alsirafy, MBBCh, MSc, MD, DipPallMed; alsirafy@kasralainy.edu.eg. Disclosures: The authors have no disclosures. JCSO 2016;13:117-121. (2016 Frontline Medical Communications. doi:10.12788/jcso.0178. 
among Yemeni cancer patients and examine how often common symptoms are missed when the assessment is limited to VR alone.

\section{Methods}

The prevalence and severity of symptoms was prospectively assessed in 50 cancer patients who were treated at cancer center. Two methods were used for the assessments, VR and SA. For the VR assessment, we asked the patients an open question about the symptoms they had and asked them to name the most distressing one. For the SA, we asked the patients about 20 physical symptoms that are known to be commonly experienced by cancer patients. ${ }^{1}$ The 20 symptoms were anorexia, bleeding, constipation, cough, diarrhea, dry mouth, dysphagia, dyspnea, early satiety, edema, fatigue/weakness, hoarseness of voice, nausea, pain, skin symptoms, sore mouth, taste changes, urinary symptoms, vomiting, and weight loss.

The severity of symptoms was graded using a 4-item verbal rating scale (None, Mild, Moderate, or Severe). For symptoms that were reported on VR and SA, the VR entry was the one taken into consideration.

Categorical variables were presented as percentages and continuous variables as mean and the standard deviation (SD). When appropriate, paired sample $t$ test or independent sample $t$ test was used to test the significance of difference between 2 continuous variables. The Pearson chisquare test was used to test the significance of difference between categorical variables.

For statistical analysis we used the Statistical Package for the Social Sciences, version 14.0. (SPSS Inc, Chicago, Illinois). A $P$ value $<.05$ was considered significant.

\section{Results}

Most of the patients were women (62\%) and most of the total number of patients were treated in the outpatient setting (72\%; Table 1$)$. In addition to the 20 symptoms that were assessed as part of the SA, another 7 physical symptoms were voluntary reported by the patients and counted for a total of 15 symptom entries. Those symptoms and their severity and prevalence among the 50 patients were: fever (mild in 4\%, moderate in 4\%), swelling (mild in 6\%, severe in $2 \%$ ), abdominal discomfort (moderate in $2 \%$, severe in $2 \%$ ), heartburn (moderate in $2 \%$, severe in $2 \%$ ), dizziness (mild in 2\%), inability to move legs (severe in 2\%), and numbness (mild in 2\%). The 7 voluntarily reported symptoms were not included in further analysis.

In all, a total of $375 \mathrm{VR}$ and SA symptom entries related to the 20 investigated symptoms. Of that total, VR accounted for $66(18 \%)$ of those entries. SA detected a further 309 (82\%) entries. The mean number of symptoms per patient was 1.3 (SD, 9.4) using VR alone, and 7.5 (SD, 3.5) using VR plus SA $(P<.001)$. The median number of symptoms per patient was 1 (range, $0-4$ ) using VR alone, and 7

TABLE 1 Patient characteristics $(\mathrm{N}=50)$

\section{Characteristic}

Median age, y (range):
$50(20-76)$

$50(20-76)$

\begin{tabular}{lr} 
Sex & $31(62)$ \\
\hline Female & $19(38)$ \\
Male & $10(20)$ \\
Primary cancer & $7(14)$ \\
Breast & $6(12)$ \\
Colorectal & $12(24)$ \\
Head and neck & $3(6)$ \\
Hematological & $12(24)$ \\
Ovary & $14(28)$ \\
Other & $36(72)$ \\
Setting of assessment & \\
Inpatient & \\
Outpatient &
\end{tabular}

(range, 0-16) using VR plus SA.

The prevalence of symptom entries that were missed with VR but found with SA was $91.4 \%$ (148/162) for mild symptoms, $75.2 \%$ (85/113) for moderate symptoms and $76 \%(76 / 100)$ for severe symptoms (Figure 1). The most common VR symptoms were pain (56\%), fatigue/weakness (12\%), vomiting (10\%), cough (10\%), and nausea (10\%). The most common symptoms using VR and SA were fatigue/weakness (78\%), pain (68\%), weight loss (62\%), anorexia (58\%), and taste changes (52\%; Figure 2).

Symptoms were of moderate or severe degree in $79 \%$ $(52 / 66)$ of VR symptoms and in 52\% (161/309) of SA symptoms $(P<.001)$. The detailed prevalence of individual symptoms according to severity using the 2 methods of assessment is shown in Table 2.The probability of not detecting symptoms when VR alone was used to assess the symptom burden was $100 \%$ for bleeding (7/7), constipation (14/14), early satiety (14/14), hoarseness (7/7), taste changes (26/26), and weight loss (31/31). It was $97 \%$ (28/29) for anorexia, 92\% (12/13) for skin symptoms, 91\% (21/23) for dry mouth, 89\% (16/18) for edema, 88\% (15/17) for dyspnea, $88 \%$ (15/17) for sore mouth, 85\% (33/39) for fatigue/weakness, $80 \%$ (8/10) for diarrhea, $80 \%(8 / 10)$ for dysphagia, 76\% (16/21) for nausea, 75\% (15/20) for cough, $75 \%$ (9/12) for urinary symptoms, $62 \%$ (8/13) for vomiting, and $18 \%(6 / 34)$ for pain.

The most distressing symptoms were pain ( $42 \%$ of patients), cough (6\%), fever (6\%), vomiting (6\%), abdominal discomfort (4\%), fatigue/weakness (4\%), nausea (4\%), sore mouth (4\%), dyspnea (2\%), dry mouth (2\%), edema $(2 \%)$, heart burn (2\%), numbness (2\%), and swelling (2\%). 


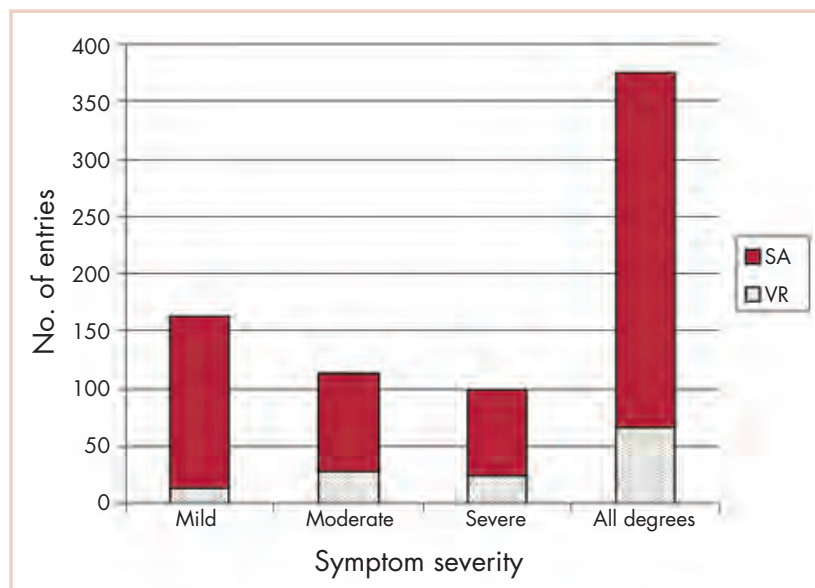

FIGURE 1 The contribution of voluntary reporting and systematic assessment to symptom entries according to the severity of symptoms.

SA, systematic assessment; VR, voluntary reporting

After excluding pain-related entries, the mean number of missed symptoms was significantly higher among patients with moderate/severe pain than those with no/mild pain (7.4 vs 5 symptoms/patient, respectively; $P=.004$ ). The mean number of missed symptoms did not differ significantly according to age ( $<50$ vs $\geq 50$ years), sex, or the setting of assessment $(P=.731, .642$, and .137 , respectively).

\section{Discussion}

Missing common symptoms experienced by cancer patients may have a negative impact on their quality of life, especially when the focus of care is symptom control. Health care professionals are encouraged to use validated assessment tools regularly to assess common symptoms. However, as in our setting, symptom burden may be assessed using VR alone. Although it is expected that SA is likely to identify more symptoms than VR would, there is not much knowledge about the pattern and the extent to which VR misses common symptoms.

The current results further support the evidence that SA is better than VR for the assessment of symptom burden. ${ }^{9-11}$ In our study, $82 \%$ of symptom entries were missed using VR alone and were detected only with SA. The median number of symptoms per patient found by SA was 7 times higher than that identified using VR. These results conform to other studies that have found that symptoms are often missed when VR is used alone. ${ }^{9-11}$ In a study conducted by Homsi and colleagues, VR and a 48-symptom SA were used to assess the symptom burden in 200 patients of whom $90 \%$ were cancer patients. ${ }^{9}$ The investigators found that VR missed $86 \%$ of symptom entries and the median number of SA symptoms was 10 times higher than that for VR symptoms. ${ }^{9}$

In another study, White and colleagues retrospectively

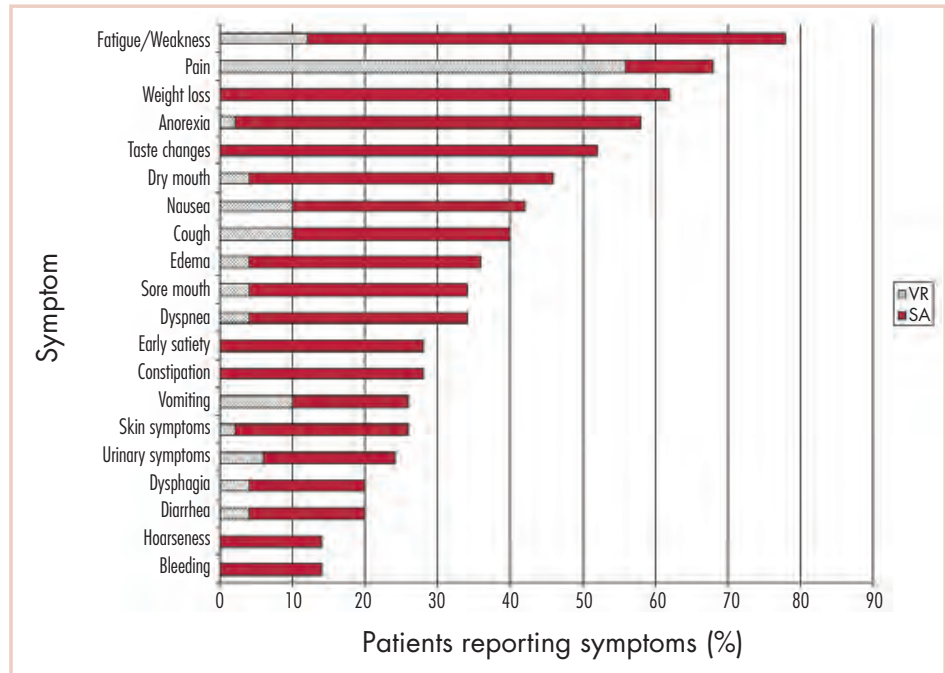

FIGURE 1 The prevalence of individual symptoms and the contribution of voluntary reporting and systematic assessment to their entries.

$\mathrm{SA}$, systematic assessment; VR, voluntary reporting

compared the self-reporting of symptoms with the systematic questioning of 38 symptoms in 47 palliative care patients, of whom $96 \%$ had a cancer diagnosis. ${ }^{10}$ They reported that $66 \%$ of symptom entries were not voluntary reported and the mean number of symptom entries using systematic questioning was higher than VR (8 vs 4 symptoms/patient). ${ }^{10}$ Jonsson and colleagues systematically assessed 41 symptoms in 62 patients with chronic noncancer pain and the spontaneous reporting of symptoms. ${ }^{11}$ The mean number of symptoms per patient found on SA was significantly higher than that for spontaneously reported symptoms ( 9.9 vs 1.3 , respectively; $P<.001) .{ }^{11}$

The consistent available evidence that most of the symptoms experienced by cancer patients are not reported voluntarily illustrates the necessity of the systematic assessment of symptoms to optimize their management. This is especially true for symptoms that are quite common among cancer patients and are largely unreported voluntarily. For example, fatigue/weakness, weight loss, anorexia, taste changes, dry mouth, and nausea were encountered in fewer than $40 \%$ of the patients included in our study. However, those symptoms were not voluntarily reported at all or reported voluntarily by a few patients. These findings are consistent with those of other studies. ${ }^{9}, 10$

The likelihood to report symptoms voluntarily may be influenced by some factors. ${ }^{9,10}$ In the current study, the likelihood of reporting symptoms voluntarily was significantly associated with the severity of symptoms $-91 \%$ of mild symptoms were not voluntary reported, compared with $76 \%$ for moderate/severe symptoms. This conforms to the results of Homsi and colleagues, who found that mild symptoms were less likely to be reported voluntarily. ${ }^{9}$ Missing mild symptoms that have not been voluntarily reported means 
TABLE 2 Prevalence and severity of 20 common physical symptoms as detected by voluntary reporting and systematic assessment ( $\mathrm{N}=50$ )

Mild, n (\%)

Moderate, $\mathbf{n}(\%)$

Severe, $\mathbf{n}(\%)$

\begin{tabular}{|c|c|c|c|c|c|c|c|c|c|}
\hline Symptom & VR & SA & All & VR & SA & All & VR & SA & All \\
\hline Anorexia & 0 & $10(20)$ & $10(20)$ & 0 & $13(26)$ & $13(26)$ & $1(2)$ & 5 (10) & $6(12)$ \\
\hline Bleeding & 0 & $4(8)$ & $4(8)$ & 0 & $3(6)$ & $3(6)$ & 0 & 0 & 0 \\
\hline Constipation & 0 & $7(14)$ & $7(14)$ & 0 & $3(6)$ & $3(6)$ & 0 & $4(8)$ & $4(8)$ \\
\hline Cough & $1(2)$ & $12(24)$ & $13(26)$ & $2(4)$ & $3(6)$ & $5(10)$ & $2(4)$ & 0 & $2(4)$ \\
\hline Diarrhea & 0 & $7(14)$ & $7(14)$ & $1(2)$ & $1(2)$ & $2(4)$ & $1(2)$ & 0 & $1(2)$ \\
\hline Dry mouth & 0 & $10(20)$ & $10(20)$ & $2(4)$ & $6(12)$ & $8(16)$ & 0 & $5(10)$ & $5(10)$ \\
\hline Dysphagia & 0 & $4(8)$ & $4(8)$ & $1(2)$ & $1(2)$ & $2(4)$ & $1(2)$ & $3(6)$ & $4(8)$ \\
\hline Dyspnea & 0 & $9(18)$ & $9(18)$ & 0 & $5(10)$ & $5(10)$ & $2(4)$ & $1(2)$ & $3(6)$ \\
\hline Early satiety & 0 & $5(10)$ & $5(10)$ & 0 & $5(10)$ & $5(10)$ & 0 & $4(8)$ & $4(8)$ \\
\hline Edema & $1(2)$ & $12(24)$ & $13(26)$ & $1(2)$ & $1(2)$ & $2(4)$ & 0 & $3(6)$ & $3(6)$ \\
\hline $\begin{array}{l}\text { Fatigue/ } \\
\text { weakness }\end{array}$ & $1(2)$ & $3(6)$ & $4(8)$ & $2(4)$ & $11(22)$ & $13(26)$ & $3(6)$ & $19(38)$ & $22(44)$ \\
\hline $\begin{array}{l}\text { Hoarseness } \\
\text { of voice }\end{array}$ & 0 & $4(8)$ & $4(8)$ & 0 & $1(2)$ & $1(2)$ & 0 & $2(4)$ & $2(4)$ \\
\hline Nausea & $1(2)$ & $11(22)$ & $12(24)$ & $3(6)$ & $4(8)$ & 7 (14) & $1(2)$ & $1(2)$ & $2(4)$ \\
\hline Pain & $9(18)$ & $3(6)$ & $12(24)$ & $10(20)$ & $2(4)$ & $12(24)$ & $9(18)$ & $1(2)$ & $10(20)$ \\
\hline $\begin{array}{l}\text { Skin } \\
\text { symptoms }\end{array}$ & 0 & $8(16)$ & $8(16)$ & 0 & $2(4)$ & $2(4)$ & $1(2)$ & $2(4)$ & $3(6)$ \\
\hline Sore mouth & 0 & $8(16)$ & $8(16)$ & $2(4)$ & $4(8)$ & $6(12)$ & 0 & $3(6)$ & $3(6)$ \\
\hline $\begin{array}{l}\text { Taste } \\
\text { changes }\end{array}$ & 0 & $10(20)$ & $10(20)$ & 0 & $8(16)$ & $8(16)$ & 0 & $8(16)$ & $8(16)$ \\
\hline $\begin{array}{l}\text { Urinary } \\
\text { symptoms }\end{array}$ & 0 & $8(16)$ & $8(16)$ & $2(4)$ & $1(2)$ & $3(6)$ & $1(2)$ & 0 & $1(2)$ \\
\hline Vomiting & $1(2)$ & $3(6)$ & $4(8)$ & $2(4)$ & $3(6)$ & $5(10)$ & $2(4)$ & $2(4)$ & $4(8)$ \\
\hline Weight loss & 0 & $10(20)$ & $10(20)$ & 0 & $8(16)$ & $8(16)$ & 0 & $13(26)$ & $13(26)$ \\
\hline
\end{tabular}

they might not be identified early enough to be adequately controlled in patients with advanced cancer. ${ }^{6}$

We also found that age and gender did not correlate significantly with the likelihood of missing symptoms on VR. Other studies have also shown no correlation with gender, ${ }^{9,10}$ diagnosis, ${ }^{10}$ race, or Bedside Confusion Scale scores. ${ }^{9}$

Another factor that was significantly associated with underreporting of symptoms voluntarily in the current study was the presence of moderate/severe pain. This may be of special significance in Yemen, where pain is largely inadequately controlled. In addition, pain was the most common VR symptom (56\%), the most common distressing symptom (42\%), and the least underreported symptom (18\%), and it was moderate/severe in $65 \%$ of patients (22/34) with pain.

These results indicate that pain is a major cause of suffering among Yemeni cancer patients. Cancer pain control in Yemen is highly inadequate as indicated by the very low opioid consumption. ${ }^{12}$ Taking measures to improve cancer pain control in Yemen would relieve the suffering of cancer patients by alleviating pain and possibly by allowing better assessment of other symptoms.

Another important application of the current results is that it shows the high symptom burden experienced by Yemeni cancer patients. The current study is the first to address the suffering of cancer patients in Yemen by exploring, partially, its physical aspect. Like many other developing countries, palliative care and cancer pain control are the 
only realistic treatment for most cancer patients in Yemen who already present in an advanced stage. ${ }^{6}$ Yemen is currently classified along with other developing countries as having no known palliative care activity. ${ }^{13}$ Efforts should be directed to initiating palliative care models that meet the needs of cancer patients in Yemen and other developing countries.

Among the limitations of this study are that it included a relatively small number of patients and that we assessed systematically only 20 physical symptoms.

In conclusion, systematic assessment of symptoms is

\section{References}

1. Teunissen SC, Wesker W, Kruitwagen C, de Haes HC, Voest EE, de Graeff A. Symptom prevalence in patients with incurable cancer: a systematic review. J Pain Symptom Manage. 2007;34:94-104.

2. Reinke LF, Feemster LC, Backhus LM, Gylys-Colwell I, Au DH. Assessment and management of symptoms for outpatients newly diagnosed with lung cancer. Am J Hosp Palliat Care. 2014 Nov 5. pii: 1049909114557635. [Epub ahead of print]

3. Walling AM, Weeks JC, Kahn KL, et al. Symptom prevalence in lung and colorectal cancer patients. J Pain Symptom Manage. 2015;49:192-202.

4. van den Beuken-van Everdingen MH, de Rijke JM, Kessels AG, Schouten HC, van Kleef M, Patijn J. Prevalence of pain in patients with cancer: a systematic review of the past 40 years. Ann Oncol. 2007;18:1437-1449.

5. van den Beuken-van Everdingen MH, de Rijke JM, Kessels AG, Schouten HC, van Kleef M, Patijn J. Quality of life and nonpain symptoms in patients with cancer. J Pain Symptom Manage. 2009;38:216-233.

6. World Health Organization. National Cancer Control Programmes: Policies and Managerial Guidelines. 2nd ed. Geneva: World Health Organization; 2002. more informative than VR in assessing the symptom burden of cancer patients and may allow earlier identification of symptoms. Routine symptom assessment should include, at least, common symptoms that are usually unreported voluntarily. Cancer patients in Yemen experience a high symptom burden, especially pain.

\section{Acknowledgment}

Data of this study were presented at the 8th World Research Congress of the European Association for Palliative Care in Lleida, Spain, June $5-7,2014$.

7. Halboub ES, Abdulhuq M, Al-Mandili A. Oral and pharyngeal cancers in Yemen: a retrospective study. East Mediterr Health J. 2012;18:985-991.

8. Harhra NA, Basaleem HO. Trends of breast cancer and its management in the last twenty years in Aden and adjacent governorates, Yemen. Asian Pac J Cancer Prev. 2012;13:4347-4351.

9. Homsi J, Walsh D, Rivera N, et al. Symptom evaluation in palliative medicine: patient report vs systematic assessment. Support Care Cancer. 2006;14:444-453.

10. White C, McMullan D, Doyle J. "Now that you mention it, doctor ...": symptom reporting and the need for systematic questioning in a specialist palliative care unit. J Palliat Med. 2009;12:447-450.

11. Jonsson T, Christrup LL, Højsted J, et al. Symptoms and side effects in chronic non-cancer pain: patient report vs. systematic assessment. Acta Anaesthesiol Scand. 2011;55:69-74.

12. International Narcotics Control Board. Narcotic drugs 2013: estimated world requirements for 2014, statistics for 2012. New York: United Nations; 2014.

13. Connor S, Sepulveda C. Global atlas of palliative care at the end of life. Geneva: World Health Organization and Worldwide Palliative Care Alliance, 2014. 